Textures and Microstructures, Vol. 33, pp. 151-171 Reprints available directly from the publisher Photocopying permitted by license only
(C) 1999 OPA (Overseas Publishers Association) N.V.

Published by license under the Gordon and Breach Science

Publishers imprint. Printed in Malaysia.

\title{
ADVANCES IN NEUTRON DIFFRACTION FOR ENGINEERING RESIDUAL STRESS MEASUREMENTS
}

\author{
A.N. EZEILO* and G.A. WEBSTER \\ Department of Mechanical Engineering, Imperial College, London, \\ $S W 72 B X, U K$
}

The increasing awareness amongst engineers and designers, of the significance of residual stresses in influencing the useful lifetimes of engineering components, has resulted in more demanding expectations being placed on the methods used to obtain these stresses. The neutron diffraction technique is emerging as the most attractive measuring method as the residual stresses can usually be obtained non-destructively to depths of up to $40 \mathrm{~mm}$ in some common engineering materials. Although it is a relatively new technique it has been used to measure the residual stresses in a range of engineering materials introduced by a wide variety of manufacturing processes such as welding, quenching, machining, shot peening, cold hole expansion and autofrettage.

In this paper the neutron diffraction technique for non-destructive residual stress measurements will be described including methods used to validate the measurements. Precautions that should be taken in order to obtain reliable measurements are outlined. Procedures being investigated in order to produce a code of practice will be presented. A representative selection of stress distributions developed by a range of manufacturing processes is examined. Some comparisons are made with strain gauge, $\mathrm{X}$-ray and numerical predictions. It is shown how the results can be of benefit in engineering stress analysis.

Keywords: Residual stress; Neutron diffraction; Shot peening; Diffraction elastic constants

* Corresponding author. 


\section{INTRODUCTION}

The catastrophic failure of structural engineering components is unacceptable. Procedures for incorporating the stresses resulting from externally applied loads into appropriate assessment procedures are well established. Residual stresses can also influence the load bearing capacity of a component (Stacey and Webster, 1988; Webster, 1989). They are usually introduced into a component during manufacture, for example from machining, welding processes, rolling etc. A postweld heat treatment or annealing procedure may be employed to relieve detrimental tensile stresses. Because most engineering failures initiate at the surface of a component as a result of higher stresses, manufacturing defects, or a harsh chemical environment, surface compressive residual stresses are an advantage. Several processes are now available to introduce compressive residual stresses in the near surface region and these include shot peening, autofrettage, laser cladding and cold hole expansion, for example. It is observed that in most cases lifetimes are increased.

Knowledge of the magnitudes and distribution of residual stresses in engineering components can assist in preventing catastrophic failure and also in improving performance. Determination of the magnitude and distribution of residual stresses which exist in a component can be difficult. When they are unknown and tensile they are traditionally overestimated, and ignored when they are compressive, in assessment procedures such as BSPD6493, to produce safe predictions. Current techniques used for measuring residual stresses include incremental hole drilling (Niku-Lari et al., 1985), X-ray diffraction (Noyan and Cohen, 1987), magnetic methods (Sarete et al., 1996), crack compliance (Cheng et al., 1991) and the neutron diffraction technique (Allen et al., 1985). Hole drilling is reasonably well established (ASTM E837-89) but is restricted by the maximum stress which can be measured (typically half the yield stress). X-ray diffraction is surface specific and depth profile measurements involve layer removal. The crack compliance technique involves growing a crack through a residual stress field which disturbs the original stress field. Finite element methods can be used to determine residual stresses although process modelling can be very complicated and the reliability of the calculations depends on the accuracy of the model employed. Neutrons can penetrate several 
millimetres into most engineering materials non-destructively making it very attractive. In addition the undisturbed stress field is measured.

\section{NEUTRON DIFFRACTION STRESS ANALYSIS}

\section{Fundamentals}

Diffraction strain measurements rely on the precise measurement of the $d$-spacing between suitably oriented $(h k l)$ planes in a polycrystal. Because $d$-spacing variations occur along different length scales in a component (Hauk, 1987), residual stresses can be classified into types 1 , 2 or 3. Type 1 or macro-residual stresses average over a number of grains and vary over dimensions comparable to those of a component. Type 2 stresses are average stresses in a single grain, while type 3 stresses are those that vary within a single grain. Types 2 and 3 stresses are usually referred to as microstresses. In general for engineering calculations only type 1 stresses are required in failure assessment procedures as these are the stresses that are traditionally used in design. However there is growing awareness of the importance of type 2 and 3 stresses as these can cause cracking to initiate as a result of local discontinuities in stress and strain. The scattered intensities attainable with neutrons usually mean that only type 1 stresses can be measured directly although a technique is available (Reimers et al., 1992) for direct measurement of type 2 stresses in large grained material. In this paper only measurements of type 1 stresses are presented.

For a monochromatic beam of wavelength $\lambda$, impinging on a material the Bragg equation describes the angular positions of the maxima for a family of crystallographic planes of separation $d$ by

$$
m \lambda=2 \theta_{(h k l)} \sin \theta_{(h k l)}
$$

where $2 \theta_{(h k l)}$ is the diffraction angle and $m$ is an integer. In a neutron diffraction experiment, the beam is masked and the lattice strain $\varepsilon_{(h k l)}$ averaged over the gauge volume in the direction of the $\mathbf{Q}$-vector (Fig. 1), is obtained from

$$
\varepsilon_{(h k l)}=\frac{\Delta d_{(h k l)}}{d_{0}}=-\Delta \theta_{(h k l)} \cdot \cot \theta_{(h k l)}
$$




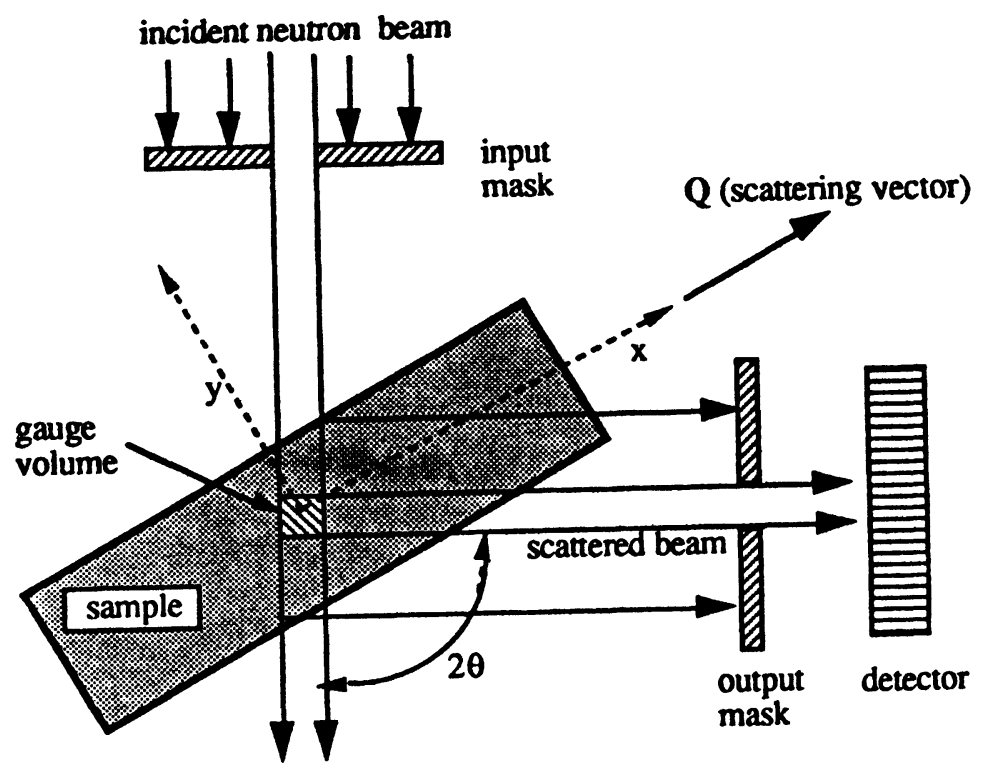

FIGURE 1 Masking of neutron beam to identify gauge volume.

To define the stress at a point completely, measurements in six orientations are required. However, when the principal directions are known, three will suffice. To convert the measured strains into engineering stresses Diffraction Elastic Constants (DECs) are required. For principal directions $x, y$ and $z$, with DECs $E_{(h k l)}$ and $\nu_{(h k l)}$, the $x$-direction principal stress is obtained from

$$
\sigma_{x}=\frac{E_{(h k l)}}{\left(1+\nu_{(h k l)}\right)\left(1-2 \nu_{(h k l)}\right)}\left[\left(1-\nu_{(h k l)}\right) \varepsilon_{x}+\nu_{(h k l)}\left(\varepsilon_{y}+\varepsilon_{z}\right)\right],
$$

and similarly for the $y$ and $z$ directions. Several precautions are needed to make reliable determinations of stress. The unstrained material lattice spacing $d_{0}\left(2 \theta_{0}\right)$ must be known to calculate strain. Also a sufficiently small gauge volume must be defined in the presence of stress gradients for adequate resolution. This can be achieved by masking the beam. In addition measurements must be made on crystallographic planes which represent bulk behaviour and which produce an intense diffraction peak at an angle $2 \theta$ close to $90^{\circ}$ so that an approximately square cross-section sampling volume is produced. 
Further detail about how to obtain accurate measurements are given in (Webster, 1992).

An alternative approach to having a monochromatic beam impinge on a sample is to use an intermittent white beam composed of a range of neutron wavelengths (velocities). The time it takes for neutrons to travel the distance $L$ from a moderator to the detector after scattering from the sample is

$$
t=\left(\frac{\lambda m L}{h}\right),
$$

where $m$ is the neutron mass and $h$ is Planck's constant. Shorter wavelength neutrons will clearly reach the detector first. The time at which neutrons reach a detector at a fixed angle $2 \theta$ can be measured. This is called Time of Flight (TOF) diffraction. Keeping $2 \theta$ fixed and differentiating the Bragg equation, for $m=1$ gives

$$
\Delta \lambda=2 \sin \theta \Delta d,
$$

or by combining Eqs. (4) and (5),

$$
\frac{\Delta \lambda}{\lambda}=\frac{\Delta d}{d}=\frac{\Delta t}{t}=\varepsilon .
$$

It is possible therefore from Eq. (4) to obtain a profile of neutron intensity vs time or wavelength. Hence a shift $\Delta t$ in the diffraction peak can be used to obtain strain measurements. Strains are converted to engineering stress using Eq. (3). TOF techniques are typically used in pulsed neutron sources. Because the full diffraction pattern is obtained simultaneously, profile refinement techniques may be used to obtain engineering strain. Here, rather than measure the shift of a single peak, the whole diffraction pattern is analysed to produce an overall lattice parameter. This can be compared with the lattice parameter for unstressed material to give the engineering strain. The same problems are encountered in establishing a stress free reference as when using a monochromatic beam.

\section{Reference $d$-spacing}

Strains can only be determined if the measured $d$-spacing is compared with that of the unstrained material $\left(d_{0}\right)$. For homogenous material a 
single value can be used while for inhomogeneous or plastically deformed material precautions must be taken. There are a variety of methods available for obtaining $d_{0}$. Measurements can be made in a powder sample of the component, in an annealed "unstressed" cube of the material, by measuring at a corner of the component or other region of low stress or by performing a force/moment-balance calculation across a section. Priesmeyer et al. (1994) proposed a technique to calculate $d_{0}$ from knowledge of the chemical composition using "Vergards law". Many of these have been tried and tested some giving variations in $d_{0}$ outside of the experimental error (Bourke, 1990). In components which exhibit a chemical gradient (welds) some workers have measured the $d$-spacing in small cubes cut from the weld region (Winholtz and Krawitz, 1994). In plastically deformed material the issue of intergranular stresses arises as discussed below.

\section{Diffraction Elastic Constants (DECs)}

Single crystals usually exhibit anisotropy described by the Single Crystal Elastic Constants (SCECs). Values are usually well tabulated in the literature for common metals (Brandes, 1983) as stiffnesses $\left(C_{i j}\right)$ or compliances $\left(S_{i j}\right)$. For an anisotropic 3-D system the generalised Hooke's law can be written as

$$
\sigma_{i j}=C_{i j k l} \varepsilon_{k l}, \quad \varepsilon_{i j}=S_{i j k l} \sigma_{k l} .
$$

Or in matrix notation;

$$
\sigma_{i}=C_{i j} \varepsilon_{j}, \quad \varepsilon_{i}=S_{i j} \sigma_{j} .
$$

$C_{i j k l}$ are the elastic stiffness constants relating the stresses in directions $i j$ to the strains in directions $k l$ while $S_{i j k l}$ are the elastic compliance constants relating the strains in directions $i j$ to the stresses in directions $k l$.

In a diffraction residual stress measurement the component material is usually polycrystalline with random orientation of grains and the presence of grain boundaries. Therefore different elastic constants to the SCECs are needed. Diffraction Elastic Constants (DECs), allow the $(h k l)$ strains to be related to the engineering stress in a polycrystal. 
DECs can be measured in a calibration experiment in situ in a beam (Ezeilo et al., 1992) or they can be calculated from the SCECs (because $C_{i j k l}$ and $S_{i j k l}$ are both 4th rank tensors they obey the transformation laws). In a measurement usually the assumption is one of uniform stress across all grains in the section. If the DECs are obtained by calculations some form of macroscopic averaging of the SCECs is necessary due to the random orientation of grains. To simplify the calculation a number of theories governing the transfer of stress and strain between grains have been proposed. Voigt (1928) assumed each grain to experience the same strain field (continuity of strain at the grain boundaries) resulting in a variation of stress from grain to grain. With this approach averages of the Young's modulus $E$ and Poisson's ratio $\nu$ for a cubic crystal are given by (Hilley et al., 1971):

$$
\begin{aligned}
& E=\frac{3\left(C_{11}+2 C_{12}\right)\left(C_{11}-C_{12}+3 C_{44}\right)}{5\left(C_{11}+2 C_{12}\right)+\left(C_{11}-C_{12}+3 C_{44}\right)}, \\
& \nu=\frac{-2\left(C_{11}-C_{12}+3 C_{44}\right)-5\left(C_{11}+2 C_{12}\right)}{4\left(C_{11}-C_{12}+3 C_{44}\right)+10\left(C_{11}+2 C_{12}\right)} .
\end{aligned}
$$

In contrast, Reuss (1924) assumed each grain experienced the same stress field (continuity of stress at the grain boundaries) resulting in a variation of strain from crystallite to crystallite. For cubic crystals,

$$
\begin{aligned}
E_{h k l} & =\frac{1}{S_{11}-2 S A_{h k l}}, \quad \nu_{h k l}=\frac{-\left(S_{12}+2 S A_{h k l}\right)}{S_{11}-2 S A_{h k l}}, \\
A_{h k l} & =\frac{h^{2} k^{2}+h^{2} l^{2}+l^{2} h^{2}}{h^{2}+k^{2}+l^{2}} .
\end{aligned}
$$

$A_{h k l}$ is the anisotropy factor describing the degree of anisotropy in a crystal, and $S$ is given by $S=\left(S_{11}-S_{12}-S_{44}\right) / 2$. The Voigt and Reuss estimates described above neglect the interaction of a grain with its neighbours. Kroner (1958), however, considers this interaction to obtain the strain and stress distributions in an anisotropic inclusion surrounded by an isotropic matrix. A coupling term $t$ is included in Eq. (7) which takes into account the grain boundary interaction stresses:

$$
\varepsilon_{i}=\left(S_{i j}+t_{i j}\right) \sigma_{j}=r_{i j} \sigma_{j}
$$


TABLE I Diffraction elastic constants for a nickel base superalloy

\begin{tabular}{|c|c|c|c|c|c|c|}
\hline \multirow[t]{2}{*}{$(h k l)$} & \multicolumn{5}{|c|}{ Diffraction elastic constant $(\mathrm{GPa})$} & \multirow{2}{*}{$\begin{array}{c}\sigma_{\mathrm{IG}} \\
(\mathrm{MPa})\end{array}$} \\
\hline & Reuss & Voigt & Kroner & $\begin{array}{c}\text { Average of } \\
\text { Reuss and Voigt }\end{array}$ & Neutrons & \\
\hline (111) & 301 & 239 & 261 & 270 & 267 & 58 \\
\hline (200) & 133 & 239 & 181 & 186 & 176 & 180 \\
\hline (220) & 230 & 239 & 235 & 235 & 243 & -96 \\
\hline (311) & 181 & 239 & 211 & 210 & 225 & 71 \\
\hline \multicolumn{6}{|c|}{ Profile refinement estimate of the elastic constant 232} & 52 \\
\hline
\end{tabular}

$t_{i j}$ can be calculated from the SCECs. The elastic constant for the Kroner model is defined by $r_{i j}$. The complete expressions which allow for the determination of $r_{i j}$ are complicated and can be found in (Dolle, 1979). Voigt, Reuss and Kroner calculations for HCP crystals can be found in (Evenschor and Hauk, 1975; Prummer et al., 1988).

One can hence use the Reuss, Voigt or Kroner estimates to calculate the DECs from the SCECs although the Kroner model agrees more closely with measurements. Voigt and Reuss are extremes to the true state of stress and strain and in reality a combination of these states must exist for continuity at the grain boundaries. A simpler and yet quite accurate approach to the Kroner model can be adopted by simply averaging the Reuss and Voigt estimates (Neerfeld, 1942). Table I shows the DECs and intergranular stresses $\left(\sigma_{\mathrm{IG}}\right)$ obtained from experiments and calculations on a nickel base superalloy. The experiments were performed on PEARL at ISIS (UK) using a stress rig in situ while the calculations were made using the SCECs of pure nickel (Ezeilo et al., 1992).

\section{Intergranular Stresses}

Residual stresses are usually generated by inhomogeneous elastic or plastic deformation. Plastic deformation involves permanent distortion of grains which introduce mismatch with the surrounding matrix at the grain boundaries since plastic strain differs from one grain to another. The grains are hence subjected to different elastic deformations produced by incompatibilities at the grain boundaries. These stresses are 
called intergranular (or type 2) stresses and can be large enough to produce significant neutron peak shifts in the absence of type 1 stresses. Some $(h k l)$ reflections can develop significant intergranular stresses after plastic deformation while others show a weak build up of these stresses. Figure 2 shows loading and unloading curves for a nickel base superalloy specimen loaded in the neutron beam beyond its mechanical yield stress. It is observed that the (200) reflection develops significant intergranular stresses while the (311) and profile refinement measurements are least affected (see also Table I). In a diffraction experiment where measurements are made in plastically deformed material it is therefore important to select an $(h k l)$ reflection least sensitive to the build-up of intergranular stresses. Models exist for the prediction of intergranular stresses based on knowledge of the local stress state allowing the active slip system to be identified. Most models describe the interactions between a grain and an isotropic matrix (Baczmanski et al., 1994). If there is uncertainty as to the magnitude of the intergranular stresses developed, the $(h k l)$ reflections least sensitive should be used in a diffraction measurement.

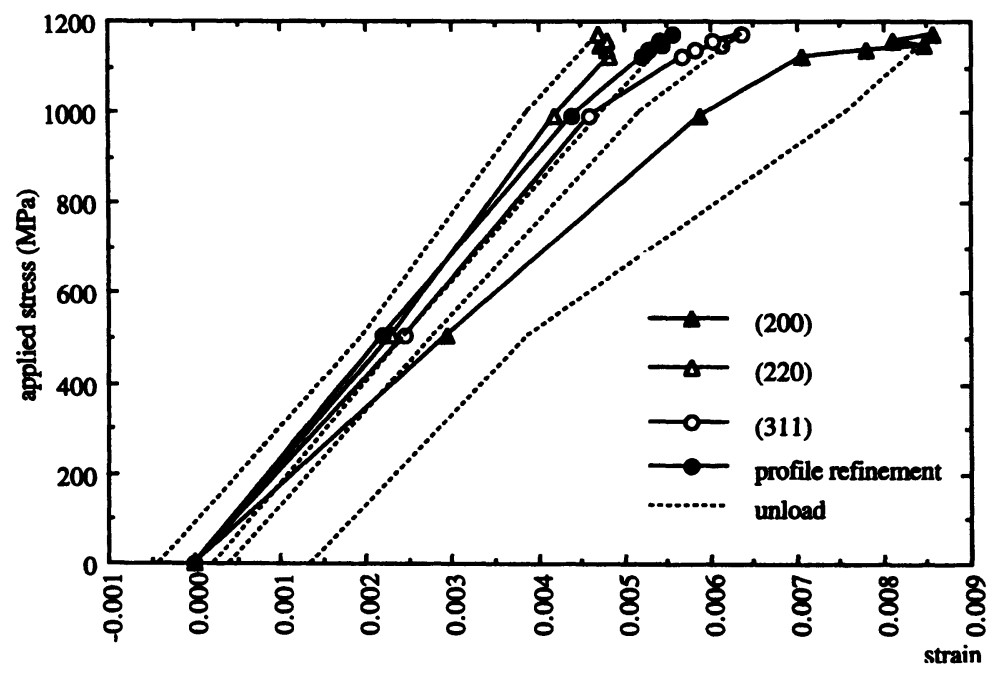

FIGURE 2 Development of intergranular strains from a tensile test in situ. 


\section{MEASUREMENT PROCEDURES}

Neutrons have been used as a probe for materials investigation for over 45 years. The use of neutrons to measure residual stress began in 1981 by Allen and co-workers at the Harwell laboratories UK (Allen et al., 1981). Since then much effort has been directed at adapting existing powder diffractometers for stress measurement purposes. Because neutrons are very attractive for non-destructive internal residual stress measurements, the technique has immense potential in industrial applications where currently used destructive techniques can involve destroying expensive components. For the technique to become accepted in industry the cost of making measurements must be competitive with the other techniques, the range of materials and applications must be broad and the procedure for making the measurements must conform to agreed procedures. The Versailles Project on Advanced Materials and Standards (VAMAS) has recently approved a Technical Working Area (TWA 20) on residual stress measurement. The aims of VAMAS are to provide a technical basis for the drafting of codes of practice and materials' specifications. TWA 20 will concentrate initially on the measurement of residual stress by neutron diffraction. Five areas of investigation have been identified and address issues such as establishing a reliable experimental procedure, determining the material characteristics which influence measurement methods, identifying the precautions needed to obtain reliable results, making comparisons with other procedures for verification purposes and developing the most appropriate analyses for extracting residual stress distributions from the neutron diffraction measurements.

Because the cost of a neutron stress measurement can be expensive a significant amount of expertise is spent developing the necessary instrumentation and procedures to reduce neutron count times thereby making the technique economical. For example a single X-ray stresswith-depth measurement will cost about $\$ 100$ while for neutrons the cost can be between $\$ 200$ and $\$ 300$. To reduce the cost of neutron measurements the approach is usually one of optimising the measuring system by either increasing the intensity of neutrons by using focusing techniques (Popovici and Yelon, 1995), improving the detector system (Lorentzen et al., 1992) or optimising the technique used (Priesmeyer et al., 1994). A few dedicated stress diffractometers have been designed 
and built over the last decade at various sites principally in Canada, Europe and USA which are optimised. Many stress measurements however, are still being performed on powder diffractometers. Position sensitive detectors (PSDs) are increasingly replacing single detectors (Lorentzen et al., 1992). Vrana et al. (1994) have used an analysing crystal in the diffracted beam path, together with a PSD to improve resolution and reduce count times for a reactor source. Reverse timeof-flight techniques achieve more efficient usage of the neutron intensity from a steady state reactor source. The peaks are measured as the derivatives of a Gaussian giving better precision over the Gaussian peak shape (Priesmeyer et al., 1994). Pulsed neutron sources are emerging as competitive alternatives to reactor sources for stress measurement. They typically supply fewer neutrons per wavelength although integrated intensities over the entire spectrum can in some cases compare well with reactors. Using the changes in lattice parameter to measure strain speeds up the measurements (Bourke, 1990). Neutron transmission measurements using the energy-dispersive neutron transmission diffraction method allow the shifts in Bragg edges to be related to engineering strain (Priesmeyer et al., 1994). Measurements can be very fast although strain measurements are averages over the transmission depth.

Materials issues always pose problems. Some austenitic steels and welds consist of large grains which are problematic for neutrons. Often the presence of texture complicates measurements and interpretation of results. Despite the difficulties successful neutron diffraction stress measurements have been made in a broad range of materials and components in the interior and close to the surface. Stresses have been measured through welds, composites, bonded joints, at surfaces in peened material etc. Winholtz and Krawitz (1994) has successfully measured the stresses in welds. Reference $d_{0}$ for the regions of varying chemical composition were obtained by making measurements on small cubes taken from these regions. Measurements have been performed on various engineering composite materials (Majumdar et al., 1991) where thermal mismatch between the composite materials produces residual stresses. Typical composites examined have been silicon carbide whisker-reinforced aluminium matrix composite, randomly oriented whisker reinforced ceramic matrix and particle-reinforced metal and ceramic matrix composites. Todd and Derby (1993) have 
applied Fourier transform techniques to deconvolute neutron intensity profiles to produce measured internal strain distribution functions in composites. A stroboscopic data collection system has been used at ISIS, UK to measure the strains induced by thermally cycling composites (Johnson, 1996). Youtsos et al. (1995) has measured residual stresses in a metal/ceramic bonded component with emphasis on the interface stresses. Stress rigs are routinely used in situ to obtain DECs. Swallowe and Osborn (1991) have analysed line shapes after uniaxial loading to large plastic strains to correlate FWHM to the change in grain size and crystal defects caused by stress. Micro-plasticity effects have been analysed using double Bragg reflections by Ono (1991) using small angle neutron scattering time-of-flight methods. Lasers are now used to assist fast neutron beam alignment and positioning of specimens. Where stress gradients are high precise definition of the gauge volume is essential. Improved specimen positioning devices have enabled specimen positioning to better than $0.1 \mathrm{~mm}$. Masking slit sizes are now as small as $0.3 \mathrm{~mm}$. Measurements of strains in through-surface regions where the gauge volume is partially immersed tend to suffer from severe instrumental surface aberrations. Webster (1992) has presented work showing geometric effects relating to most multi-detectors and PSDs. Calculations have been made which model geometric effects and allow for the necessary corrections and extraction of the real strains (Johnson, 1996).

\section{PRACTICAL APPLICATIONS}

To illustrate the application of neutron diffraction for measuring residual stress a number of practical cases will be considered. Examples involving homogeneous and inhomogeneous material and shallow and steep stress gradients will be presented, where appropriate comparisons will be made with other experimental methods and numerical analysis techniques. All neutron measurements were made on instrument D1A at the ILL in Grenoble, France with a neutron wavelength of $0.19 \mathrm{~nm}$.

\section{Shallow Stress Gradients}

When a component is chemically homogeneous and the stress gradients are not too steep a straightforward measuring approach can be 
adopted. The autofrettage of thick walled pressure piping is known to produce a shallow stress gradient. Autofrettage is used to introduce compressive residual stresses at the bore of pressure vessels, to improve resistance to fatigue failure. Residual stresses have been determined in a ring specimen taken from an autofrettaged steel cylinder using neutrons and the established Sachs boring technique for comparison (Stacey et al., 1985). A gauge volume, $1.8 \times 1.8 \times 8 \mathrm{~mm}$, was defined by masking the incident and diffracted beams. This volume was then traversed in steps for radial, axial and hoop direction strains. The (211) set of crystal planes were used. The zero strain reference position corresponding to $2 \theta=109.135^{\circ}$ was obtained by sampling a large volume of unstressed material. The residual stresses determined for the hoop direction are shown in Fig. 3. Good agreement is achieved with the Sachs boring method and with a modification of this process involving machining

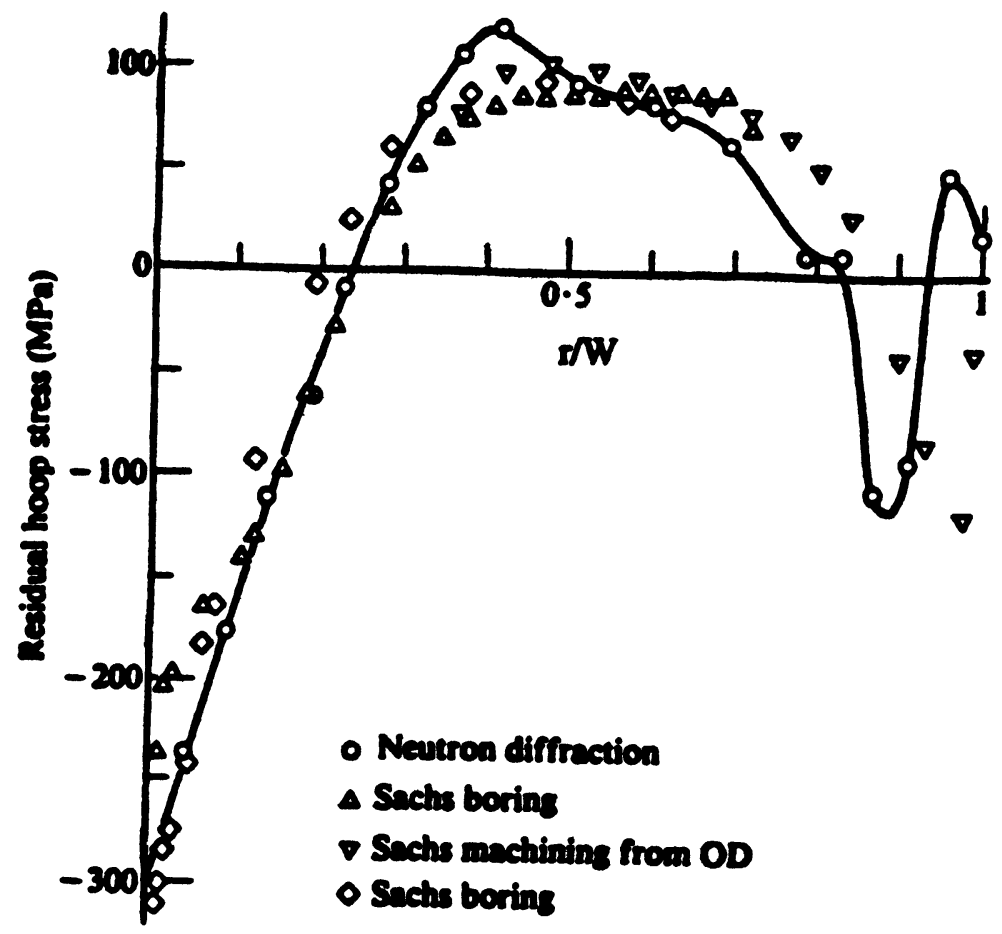

FIGURE 3 Comparison of residual hoop stresses determined by the neutron diffraction and Sachs methods. 
from the outer diameter. The high beneficial compression generated at the bore by the autofrettage treatment is clearly evident.

\section{Steep Stress Gradients}

When stress gradients are steep precise sample positioning, gauge volume definition and accurate understanding of the neutron beam optics is essential. Usually steep stress gradients occur at surfaces or at interfaces and will require the gauge volume to be partially immersed. Shot peening is a mechanical surface treatment process generating compressive residual stresses in the very near surface region resulting in improved fatigue resistance. The magnitude and distribution of these stresses must be known if a quantified assessment of fatigue life improvement is to be made. Residual stresses have been measured in a plate peened on two faces with different peening conditions, by stepping the gauge volume through the surface regions in the principal directions. A matchstick shaped gauge volume, $1 \times 1 \times 25 \mathrm{~mm}$, was used to exploit the symmetry from the peening process. The (311)

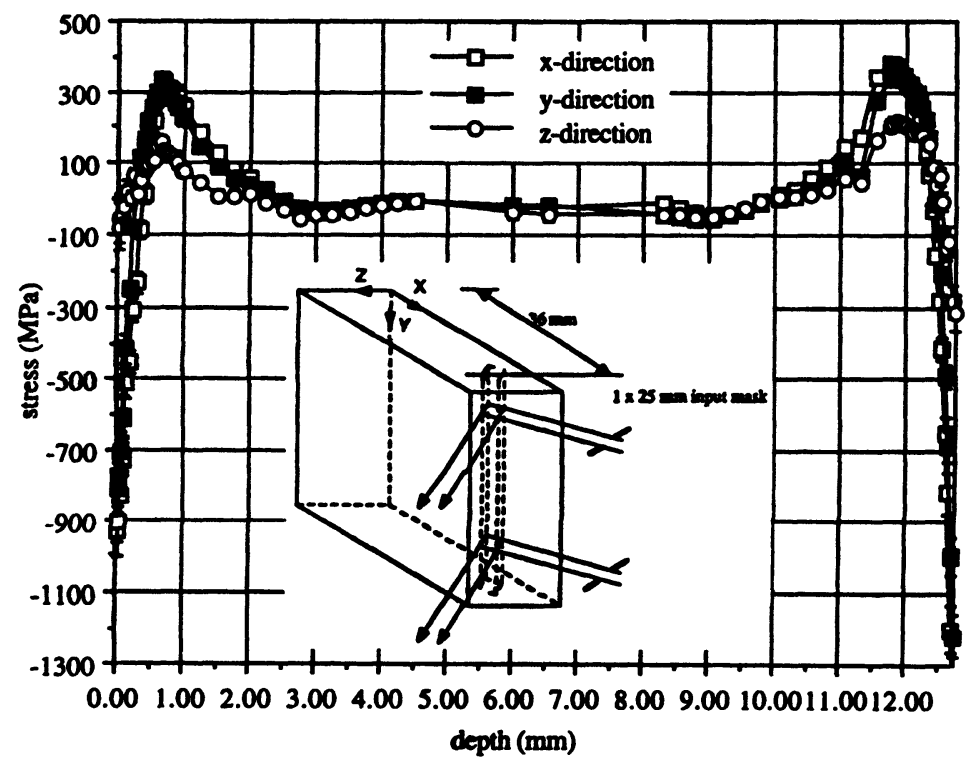

FIGURE 4 Residual stresses generated by shot peening in a Udimet 720 plate. 
crystal reflection was used, which appears to be relatively insensitive to the build-up of intergranular stresses (Ezeilo et al., 1992). It was possible to measure strains as close as $0.1 \mathrm{~mm}$ to the surface. Because the gauge volume was partially immersed the data required corrections to establish the positions of the centroid of the gauge volume. The residual stresses were then calculated. By traversing the gauge volume through both surfaces it was observed that there appeared to be no severe instrumental surface aberrations on D1A which are commonly experienced on other instruments. Measured residual stresses are shown in Fig. 4 showing compressive stresses at the surface close to the yield stress of the material. There is a rapid reduction in the compressive stresses to zero in $0.3 \mathrm{~mm}$. Also of interest are the subsurface tensile stresses measured with neutrons which are often not seen when using X-rays (Ezeilo et al., 1997). These results show the importance of neutrons in discovering subsurface stresses non-destructively.

\section{Inhomogeneous Material}

The following example shows how residual stresses can be measured using neutrons in an inhomogeneous material by examining a laser surface remelted steel specimen. The process involves localised melting which produces a hard surface layer and generates residual stresses on cooling. A hard surface offers improved resistance to corrosion and wear whilst residual stresses influence the fatigue behaviour of the material. Either tensile or compressive residual stresses can be introduced at the surface depending on the process route adopted. Measurements have been made on a $12 \%$ chromium martensitic stainless steel after laser surface remelting to a depth of $0.23 \mathrm{~mm}$ (Ezeilo $e t$ al., 1991). A microstructural examination revealed 3 distinct layers, a $0.23 \mathrm{~mm}$ melted zone, a $0.23 \mathrm{~mm}$ intermediate heat affected zone and the remainder base material. In order to achieve high spatial resolution the neutron beam dimensions at the specimen were reduced to a matchstick shape measuring $0.33 \times 0.33 \times 25 \mathrm{~mm}$. The (211) set of crystallographic planes was used at a diffraction angle of $109^{\circ}$. Measurements were made in three orthogonal directions. As a consequence of the microstructural variations across the specimen, the value of the unstressed $d$-spacing $\left(d_{0}\right)$ had to be obtained for each microstructural region. This was achieved by obtaining the $d_{0}$ value at the surface in the 


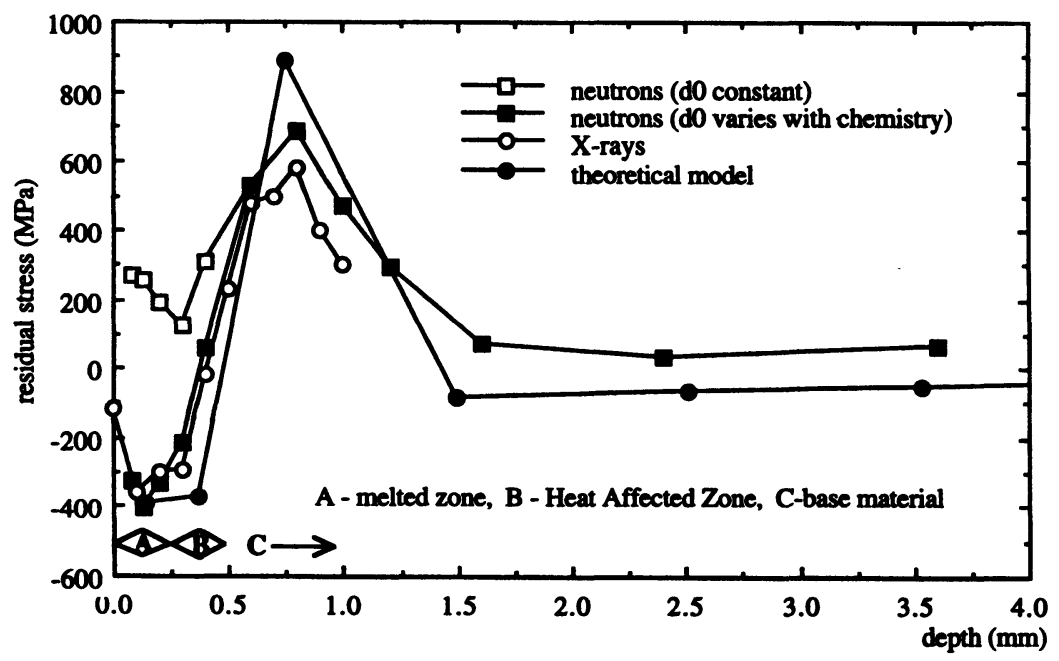

FIGURE 5 Comparison of measured and predicted residual stresses in a laser treated surface.

melted layer by assuming the normal stress at the surface to be zero. A value for $d_{0}$ was also measured in the base material. For intermediate material a linear variation of $d_{0}$ was adopted. Results are shown in Fig. 5 for in-plane ( $x$-direction) residual stresses with neutron diffraction measurements compared with X-rays and a thermo-mechanical model. Similar results were obtained in the other in-plane direction ( $y$-direction). For the set of laser processing parameters used compressive stresses are produced close to the surface although substantial tensile stresses are generated subsurface. These measurements reveal the importance of using appropriate values of the unstressed lattice spacing in materials with varying microstructures in neutron diffraction stress calculations.

\section{REDISTRIBUTION OF RESIDUAL STRESSES}

\section{Low Cycle Fatigue}

As described earlier shot peening introduces beneficial compressive residual stresses at the surface of a component enhancing fatigue performance. It is known that residual stresses can be redistributed and in 
some cases totally relaxed, after experiencing external loading (thermal or mechanical) (Ezeilo et al., 1994). In order to investigate the effect of Low Cycle Fatigue (LCF) on the residual stress state in a peened plate measurements were made before and after fatigue cycling at different strain amplitudes. A nickel base superalloy was examined measuring on the $(311)$ crystal reflection at $123^{\circ} 2 \theta$. The material had been peened using identical conditions on both faces and subjected to cyclic loading in four point bending. A $1 \times 1 \times 20 \mathrm{~mm}$ gauge volume was used following the procedure described earlier for steep stress gradients. Measurements were made before and after fatigue cycling. Figure 6 compares the longitudinal ( $x$-direction) residual stress distributions near the surface of the specimen prior to fatigue loading, after 5 cycles at a strain amplitude of $0.6 \%$, after 161,000 cycles at $0.61 \%$ and after 36,000 cycles at $1.13 \%$. The static yield strain for the material is about $0.45 \%$. Results shown are for the face which had experienced tensile fatigue loading. It is seen that most of the redistribution of residual stress occurs in the first few cycles for the $0.6 \%$ strain amplitude. For large strain amplitudes the residual stress is significantly redistributed. Measurements were also made on the face which had experienced compressive fatigue loading showing a more rapid relaxation of residual stresses. These measurements illustrate the ability of the neutron

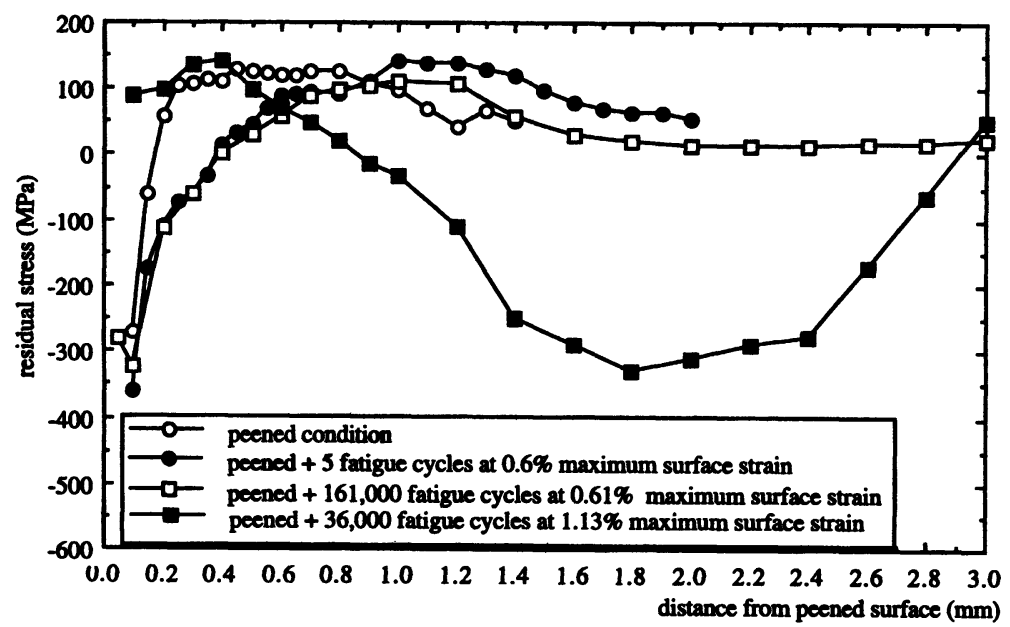

FIGURE 6 In-plane shot peening residual stresses before and after fatigue cycling. 
diffraction technique to measure the evolution of a residual stress state and determine the effectiveness of a shot peening operation.

\section{High Temperature Soaking}

Residual stress fields may be altered by sustained loading at elevated temperatures. An investigation was carried out to measure the residual stresses in a C-shaped specimen geometry made of a low alloy steel chosen to represent the behaviour of a pressurised cylinder. Stresses were introduced by pre-tensioning. Residual stresses were measured before and after soaking at $550^{\circ} \mathrm{C}$ for $250 \mathrm{~h}$. Figure 7 shows the measured hoop stress before and after soaking and also predictions from finite element calculations after pre-tensioning the ring. The results show that soaking of a low alloy steel at normal operating temperature can cause relaxation of an initially introduced residual stress field. This example demonstrates that neutron diffraction can be employed to investigate the influence of heating in modifying residual stress distributions.

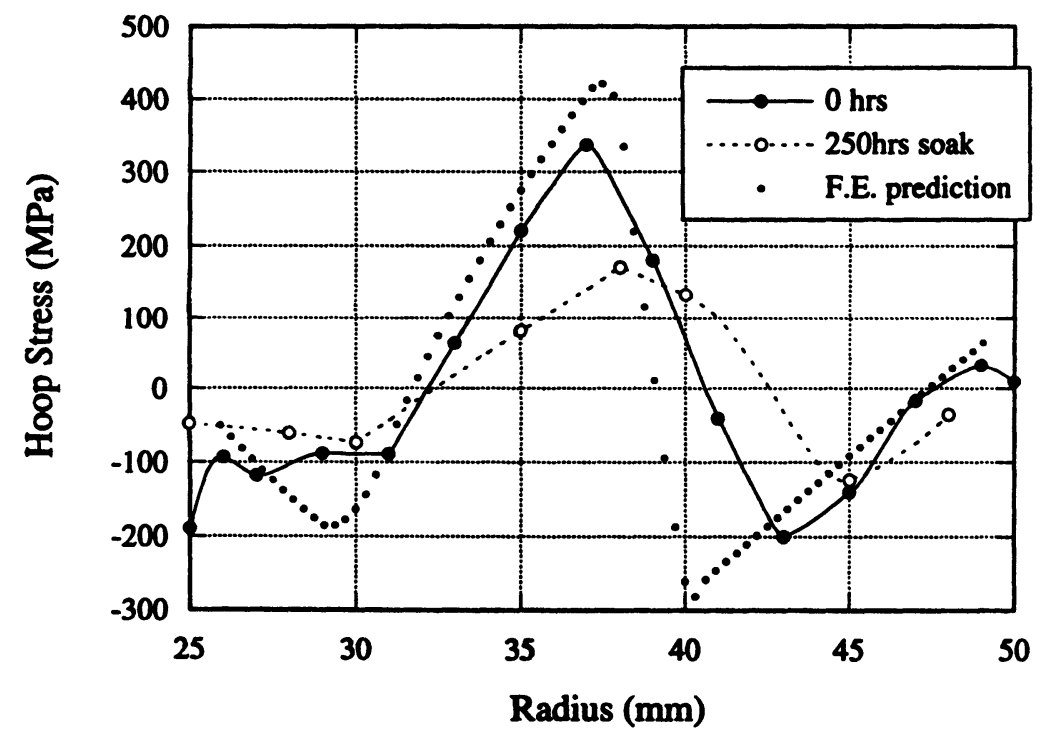

FIGURE 7 Effect of a high temperature soak on a residual stress distribution. 


\section{CONCLUSIONS}

The neutron diffraction technique has been presented as an extremely attractive tool for the determination of stresses within the interior of engineering components non-destructively. Developments in hardware which have led to more efficient use of neutrons have been discussed. Examples have been presented of measurements of residual stresses resulting from a variety of manufacturing processes and in-service loading conditions. Precautions necessary for making reliable measurements and the progress made towards establishing a protocol for making measurements have been discussed.

\section{References}

Allen, A., Andreani, C., Hutchings, M.T. and Windsor, C.G. (1981). Measurement of internal stress within bulk materials using neutron diffraction. NDT Int., 249-254.

Allen, A.J., Andreani, C., Hutchings, M.T. and Windsor, C.G. (1985). Neutron diffraction methods for the study of residual stress fields. Adv. Phys., 34, 445-473.

Baczmanski, A., Wierzbanowski, K., Lipinski, P., Helmholdt, R.B., Ekambaranathan, G. and Pthiraj, B. (1994). Examination of the residual stress field in plastically deformed polycrystalline material. Phylosophical Magazine A, 69(3), 437-449.

Bourke, M.A.M. (1990). Residual stress measurement in engineering components by neutron diffraction. PhD Thesis, University of London.

Brandes, A.E. (1983). Smithells Metals Reference Book, 6th edn. Butterworths and Co. Ltd, London.

Cheng, W., Finnie, I. and Vardar, O. (1991). Measurement of residual stresses near the surface using the crack compliance method. J. Engineering Materials and Technology, 113, 199-204.

Dolle, H. (1979). The influence of multiaxial stress states, stress gradients and elastic anisotropy on the evaluation of (residual) stresses by X-rays. J. Appl. Cryst., 12, 489-501.

Evenschor, P.D. and Hauk, V. (1975). Z Metallkde, 66, 210-213.

Ezeilo, A.N., Webster, G.A., Webster, P.J., Roth, M. and Muster, W.J. (1991). Comparison of X-ray and neutron diffraction determinations of residual stresses in a laser treated martensitic steel. Proc. of the 2nd European Conf. on Adv. Materials and Processes (Eds. Clyne, T.W. and Withers, P.J.), Euromat 91, Cambridge, 3, 389-394.

Ezeilo, A.N., Webster, G.A., Webster, P.J. and Wang, X. (1992). Characterisation of elastic and plastic deformation in a nickel superalloy using pulsed neutrons. Physica $B, 180$ \& 181, 1044-1046.

Ezeilo, A.N., Webster, G.A., Webster, P.S. and Webster, P.J. (1994). Influence of cyclic loading on residual stress distribution caused by cold hole expansion. Proc 4th Int. Conf. on Residual Stress, Baltimore, USA, Soc. Exp. Mechanics, 1275-1284.

Ezeilo, A.N., Webster, G.A., Webster, P.S. and Castex, L. (1997). Comparison of X-ray and neutron diffraction residual stress measurements in a duplex peened sample. 5 th Int. Conf. on Residual Stresses, Linkoping, Sweden.

Hauk, V. (1987). Non-destructive methods of measurement of residual stresses. Advances in Surface Treatments (Ed. Niku-Lari), Vol. 4, 251-302. Pergamon Press, Oxford.

Hilley, M.E., Larson, A.J., Jatczak, C.F. and Ricklefs, R.E. (Eds.) (1971). Residual stress measurement by X-ray diffraction. SAE Information Report J784a SAE Inc., Warrendale PA. 
Johnson, M.W. (1996). PREMIS Final Technical Report, BriteEuram II Project No. 5129. Rutherford Appleton Laboratory Technical Report RAL-TR-96-068.

Kroner, E. (1958). Berechnung der Elastischen Konstant des Vielkristalls aus den Konstant des Einkristalls. Z. Physik, 151, 504.

Lorentzen, T., Leffers, T. and Juul Jensen, D. (1992). Implementation and application of a PSD set-up for neutron diffraction strain measurements. Measurement of Residual and Applied Stresses using Neutron Diffraction (Eds. Hutchings, M.T. and Krawitz, A.D.), Kluwer Academic Publishers, Netherlands.

Majumdar, S., Singh, J.P., Kupperman, D. and Krawitz, A. (1991). Application of neutron diffraction to measure residual strains in various engineering composite materials. J. Eng. Matls and Tech., 113, 51.

Neerfeld, H. (1942). Mitt. KWI Eisenforsch., Vol. 24, 61-70.

Niku-Lari, A., Lu, J. and Flavenot, J.F. (1985). Measurement of residual stress distribution by the incremental hole-drilling method. J. Mechanical Working Technology, II, 2.

Noyan, I.C. and Cohen, J.B. (1987). Residual stress, measurement by diffraction. Materials Research Engineering, Springer-Verlag.

Ono, M. (1991). Non-destructive study on microplasticity of metallic materials by neutron double Bragg reflection at small scattering angle. Int. Conf. on Neutron Scattering, ICNS, Oxford.

Popovici, M. and Yelon, W.B. (1995). Focussing Monochromators for Neutron Diffraction. J. of Neutron Research, 3(1), 1-25.

Priesmeyer, H.G., Larsen, J. and Meggers, K. (1994). Neutron diffraction for nondestructive strain/stress measurements in industrial devices. J. of Neutron Research, 2(1), 31-52.

Prummer, R., Pfeiffer, W. and Reisacher, E. (1988). X-ray elastic constants and residual surface stress state in glass ceramic. 2nd Int. Conf. on Residual Stresses, Nancy.

Reimers, W., Crostack, H.A., Wrobel, M. and Eckold, G. (1992). Investigation of large grained samples - examples. Measurement of Residual and Applied Stresses using Neutron Diffraction (Eds. Hutchings, M.T. and Krawitz, A.D.), Kluwer Academic Publishers, Netherlands, 263-276.

Reuss, A. (1924). Berechnung der fliessgrenze von misch kristallen auf grund der plastizitatsbedingung fur einkristalle. Z. Angew, Math. Mech., 9, 49.

Sarete, J., Saquet, O. and Chicois, J. (1996). Monitoring applied or internal stresses by magnetic or micromagnetic methods. 4th European Conf. on Residual Stresses, Cluny, France.

Stacey, A., MacGillivray, H.J., Webster, G.A., Webster, P.J. and Ziebeck, K.R.A. (1985). Measurement of residual stress by neutron diffraction. J. of Strain Analysis, 20, 93-100.

Stacey, A. and Webster, G.A. (1988). Influence of residual stress on fatigue crack growth in thick-walled cylinders. ASTM Symposium "Analytical and Experimental Methods of Residual Stress Effects in Fatigue" (Eds. Champoux, R.L., Underwood, J.H. and Kapp, J.A.), ASTM STP 1004, American Society for Testing and Materials, 107-121.

Swallowe, G.M. and Osborn, J.C. (1991). The effect of stress on Bragg peak shapes in copper. Int. Conf. on Neutron Scattering, ICNS, Oxford.

Todd, R.I. and Derby, B. (1993). Deconvolution of measured internal strain distribution functions for A1203/SiCp composites from neutron diffraction results. (Eds. Barrera, V. and Dutta, I.) 147 TMS-AJME, Warrendale, PA.

Voigt, W. (1928). Lehrbuch der Kristallphysik. Leipzig: Teubner, 962.

Vrana, M., Lukas, P., Mikula, P. and Kulda, J. (1994). Bragg diffraction optics in high resolution strain measurements. Nuclear Instruments \& Methods in Physics Research $A, 338,125-131$. 
Webster, G.A. (1989). Propagation of fatigue cracks through residual stress fields. Fatigue \& Stress, (Ed. Lieurade, H.P.), IITT International, Gournay-sur-Marne, France, pp. 9-20.

Webster, P.J. (1992). Spatial resolution and strain scanning. Measurement of Residual and Applied Stresses using Neutron Diffraction (Eds. Hutchings, M.T. and Krawitz, A.D.), Kluwer Academic Publishers, Netherlands, 235-251.

Winholtz, R.A. and Krawitz, A.D. (1994). Depth probing of stress tensors using neutron diffraction. Proc. of the 4th International Conf. on Residual Stresses, Baltimore, USA.

Youtsos, A.G., Schroder, J. and Timke, T. (1995). Residual stress investigations near material interfaces by neutron diffraction at the JRC-Petten. Proc. of the 4th Int. Symposium on Advanced Composites, Corfu, Greece. 